
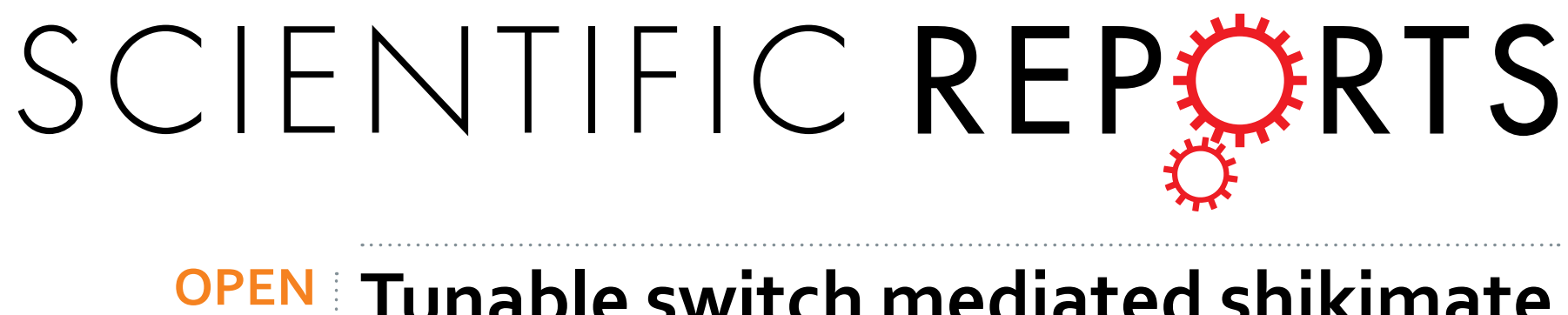

\title{
Tunable switch mediated shikimate biosynthesis in an engineered non- auxotrophic Escherichia coli
}

Received: 08 March 2016

Accepted: 23 June 2016

Published: 13 July 2016

\author{
Pengfei Gu, Tianyuan Su, Qian Wang, Quanfeng Liang \& Oingsheng Oi
}

Shikimate is a key intermediate in the synthesis of neuraminidase inhibitors. Compared with traditional methods, microbial production of shikimate has the advantages of environmental friendliness, low cost, feed stock renewability, and product selectivity and diversity. Despite these advantages, shikimate kinase I and II respectively encoded by aroK and aroL are inactivated in most shikimate microbial producers, thus requiring the addition of aromatic compounds during the fermentation process. To overcome this problem, we constructed a non-auxotrophic, shikimate-synthesising strain of Escherichia coli. By inactivation of repressor proteins, blocking of competitive pathways and overexpression of key enzymes, we increased the shikimate production of wild-type $E$. coli BW25113 to $1.73 \mathrm{~g} / \mathrm{L}$. We then designed a tunable switch that can conditionally decrease gene expression and substituted it for the original aroK promoters. Expression of aroK in the resulting P-9 strain was maintained at a high level during the growth phase and then reduced at a suitable time by addition of an optimal concentration of inducer. In 5-L fed-batch fermentation, strain P-9 produced $13.15 \mathrm{~g} / \mathrm{L}$ shikimate without the addition of any aromatic compounds. The tunable switch developed in this study is an efficient tool for regulating indispensable genes involved in critical metabolic pathways.

Shikimate, an important intermediate in the aromatic amino acid pathway, can be used as a chiral template for the synthesis of neuraminidase inhibitors such as oseltamivir (Tamiflu $\left.{ }^{\circledR}\right)^{1}$. Shikimate is also a promising building block for the synthesis of other biological compounds ${ }^{2,3}$. Because chemical synthetic strategies are restricted by environmental concerns, commercial shikimate production currently involves extraction from seeds of Illicium plants-a multistep, low-yield and costly process. To overcome these drawbacks and meet rapidly growing demand, microbial production of shikimate from renewable carbon sources has attracted increasing attention, with several microbial production strains with relatively high titre and yield having been engineered ${ }^{4,5}$. Escherichia coli, a widely used host for which engineering tools are readily available, is characterised by a clear genetic background and fast growth in inexpensive media and is thus a preferred candidate for shikimate production ${ }^{6-8}$.

In E. coli, the first step of the shikimate pathway is the condensation of phosphoenol pyruvate (PEP) and erythrose 4-phosphate (E4P) into 3-deoxy-D-arabinoheptulosonate 7-phosphate (DAHP). This conversion is carried out by DAHP synthase isoenzymes encoded by aroF, aro $G$ and $a r o H$, which are feedback-resistant by L-tyrosine, L-phenylalanine and L-tryptophan, respectively ${ }^{9}$. DAHP is then converted to shikimate via three steps catalysed by dehydroquinate synthase, 3-dehydroquinate dehydratase and shikimate dehydrogenase, respectively. Afterwards, shikimate is transformed to chorismate, a common precursor to L-phenylalanine, L-tyrosine and L-tryptophan (Fig. 1).

Metabolic engineering approaches used to construct a shikimate-producing strain mainly focus on the central carbon metabolic pathway and the shikimate pathway. Inactivation of the PEP:carbohydrate phosphotransferase (PTS) system and overexpression of $t k t A$ and $p p s A$ encoding transketolase and PEP synthase, respectively, are used to increase intracellular levels of E4P and PEP, the two precursors of shikimate ${ }^{10-12}$. These modifications are commonly combined with the plasmid-based overexpression of $\operatorname{aro} G^{F B R}$, aroB, aroD and aroE, which are crucial genes involved in the shikimate synthetic pathway ${ }^{2,6}$. To block carbon flux from shikimate into the aromatic amino acid pathway, aroK and aroL genes encoding shikimate kinase I and II are usually deleted. Recombinant E. coli strains with these genetic modifications can produce 0.27 mol shikimate per mol glucose in fed-batch fermentation ${ }^{13}$. To avoid the genetic instability of plasmids, a modified chemically induced chromosomal evolution method has been applied to integrate multiple copies of a gene cluster containing aro $G^{F B R}$, aroB, aroE and $t k t A$ 


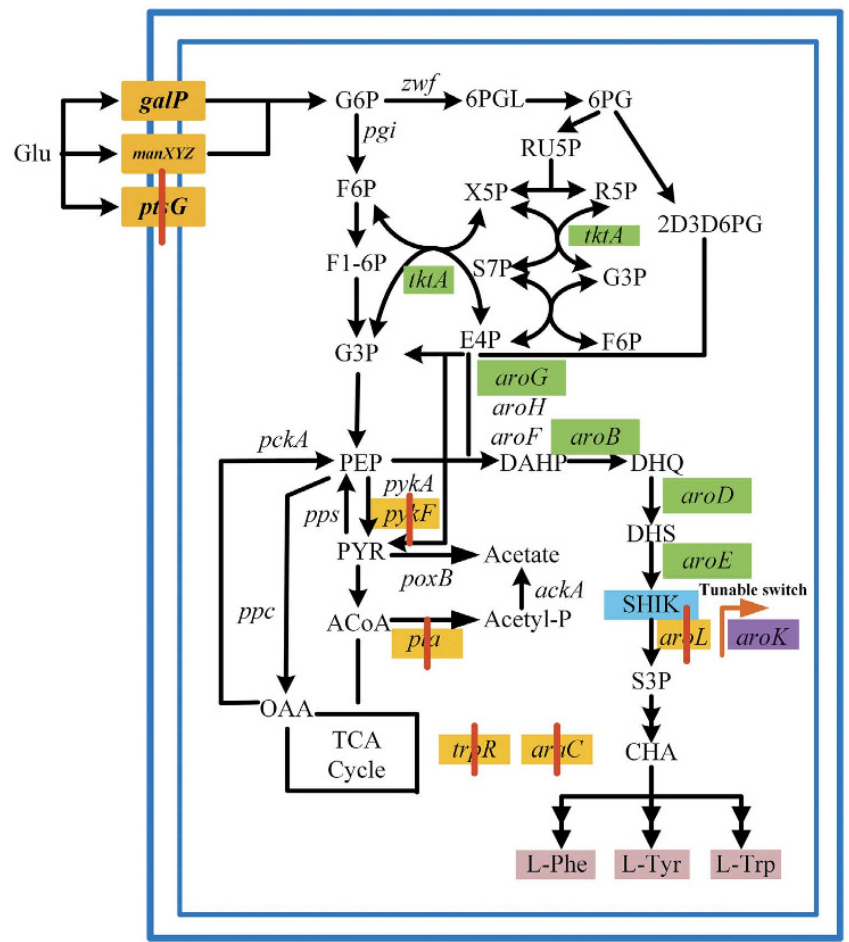

Figure 1. Strategy used to construct the shikimate-synthesising strain P-9. Green boxes represent plasmidoverexpressed genes, and red bars indicate genes that were deleted. The wild-type promoters of aroK was replaced by a tunable switch. Glu, glucose; G6P, glucose-6-phosphate; F6P, fructose-6-phosphate; F1-6P, fructose-1, 6-bisphosphate; G3P, glyceraldehyde-3-phosphate; 6PGL,6-phosphoglucono-lactone; 6PG, 6-phosphogluconate; 2D3D6PG, 2-dehydro-3-deoxy-D-gluconate-6-phosphate; RU5P, ribulose-5-phosphate; X5P, xylulose-5-phosphate; R5P, ribose-5-phosphate; S7P, sedoheptulose-7-phosphate; E4P, erythrose-4phosphate; PEP, phosphoenolpyruvate; PYR, pyruvate; ACoA, acetyl coenzyme A; OAA, oxaloacetic acid; DAHP, 3-deoxy-D-arabino-heptulosonate-7-phosphate; DHQ, 3-dehydroquinate; DHS, 3-dehydroshikimate; SHIK, shikimate; S3P, shikimate-3-phosphate; CHA, chorismate; L-Phe, L-phenylalanine; L-Tyr, L-tyrosine; L-Trp, L-tryptophan.

into the E. coli chromosome ${ }^{8}$. The final strain, E. coli SA116, can produce $3.12 \mathrm{~g} / \mathrm{L}$ of shikimate, with a yield of $0.33 \mathrm{~mol} / \mathrm{mol}$ glucose.

Because of the permanent deletion of shikimate kinase encoded by aroK and aroL, these engineered shikimate producers are auxotrophic. During fermentation, three aromatic amino acids and other aromatic compounds, such as $p$-hydroxybenzoic acid, potassium $p$-aminobenzoate and 2,3-dihydroxybenzoic acid, must therefore be added to the medium to maintain normal host growth, thus increasing the cost of industrial production ${ }^{14}$.

To overcome this problem, in this study we developed an engineered tunable switch that was applied to the controllable expression of aroK. In the recombinant strain, the expression of aro $K$ was conditionally repressed after accumulation of adequate biomass, thus demonstrating the successful generation of a non-auxotrophic, shikimate-synthesising E. coli.

\section{Results and Discussion}

Construction and characterization of a tunable switch. Because the activity of shikimate kinase I encoded by aroK is crucial for increasing shikimate accumulation and maintaining normal cell growth, a tunable switch was first constructed to allow appropriate regulation of AroK (Fig. 2). When no inducer was incorporated into the culture, the expression of TetR controlled by $\mathrm{P}_{\mathrm{BAD}}$ promoter was repressed, with consequent normal expression of the target gene under the regulation of $\mathrm{P}_{\mathrm{LtetO1} 1}$. When $\mathrm{L}$-arabinose was added to induce the $\mathrm{P}_{\mathrm{BAD}}$ promoter, $\mathrm{P}_{\mathrm{LtetO} 1}$ promoter transcription was partially repressed by expressed TetR as a function of L-arabinose concentration, thereby leading to decreased target gene expression.

To verify the function of this tunable switch, sfgfp encoding super-folding green fluorescent protein (sfGFP) was selected as a reporter. When $1.00 \mathrm{~g} / \mathrm{L} \mathrm{L}$-arabinose was incorporated into the medium at 0,4 , or $8 \mathrm{~h}$ after inoculation of seed cultures, the relative fluorescence intensities of sfGFP were all obviously decreased compared with the control strain lacking inducer (Fig. 3). When L-arabinose was added at the start of batch cultivation, fluorescence intensities were maintained at a relatively low level. At $24 \mathrm{~h}$, the fluorescence intensity of strain $0+$ was 17.83-fold lower than the control. Similarly, 11.82- and 8.63-fold reductions were observed when supplementation with L-arabinose was delayed for 4 or $8 \mathrm{~h}$, respectively. For strains $4+$ and $8+$, a delay of approximately 8-12 h occurred before fluorescence intensities reached a relatively low level. Although only a small amount of 
L-arabinose with different concentration

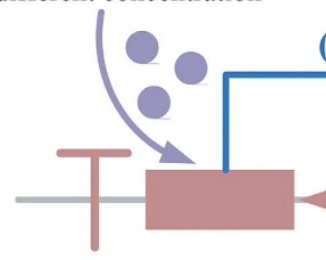

$\mathrm{T}_{\mathrm{BBa} B \text { B0014 }}$

\begin{abstract}
$\quad \mathrm{P}_{\mathrm{C}} \quad \mathrm{P}_{\mathrm{BAD}}$
\end{abstract}
$(+)$

Module 1

$(-)$

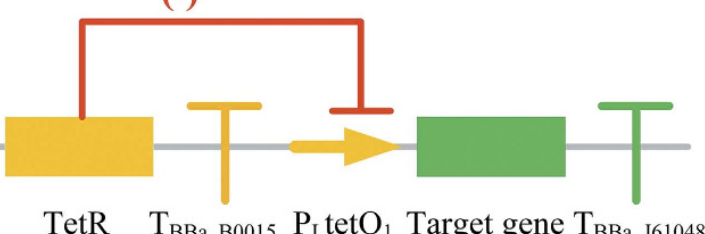

Module 2

\section{Module 3}

Figure 2. Design of the tunable switch. To simplify the overall construction process, design of the switch was divided into three modules indicated by different colours.

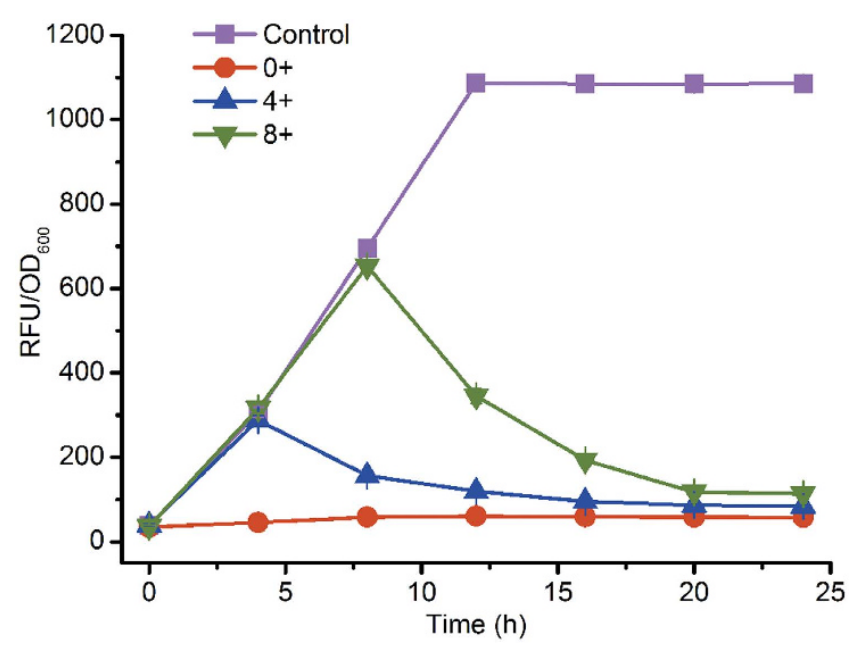

Figure 3. Characterisation of the tunable switch at different supplementation time points. Numbers $0+, 4+$ and $8+$ indicate strains after supplementation of the medium with $1.00 \mathrm{~g} / \mathrm{L} \mathrm{L}$-arabinose at 0,4 or $8 \mathrm{~h}$, respectively, after inoculation of seed cultures. Escherichia coli strains were cultured in $50 \mathrm{~mL}$ Luria-Bertani medium shaken at $250 \mathrm{rpm}$ and $37^{\circ} \mathrm{C}$. Error bars represent standard deviations based on three replicate fermentations.

new sfGFP was generated after repression of the tunable switch, time was still required for proteolysis of existing intracellular sfGFP ${ }^{15}$.

We next investigated the relationship between inducer concentration and repression level of the tunable switch. As shown in Fig. 4, relative fluorescence intensity decreased from 1381.53 to 91.52 RFU/OD 600 when the concentration of L-arabinose was increased from 0 to $2.00 \mathrm{~g} / \mathrm{L}$. Increasing the L-arabinose concentration to $4.00 \mathrm{~g} / \mathrm{L}$ did not further reduce the fluorescence intensity. Within the range of the $0-0.02 \mathrm{~g} / \mathrm{L}$ inducer, an approximate linear relationship was exhibited between the repression level of the tunable switch and L-arabinose concentration. Even at the highest repression level, achieved by the addition of $2.00 \mathrm{~g} / \mathrm{L} \mathrm{L}$-arabinose, a fluorescence intensity of $91.53 \mathrm{RFU} / \mathrm{OD}_{600}$ was still maintained. Rogers et al. have demonstrated that AraC- and TetR-inducible systems exhibit uninduced accumulation of GFP when high-copy vectors are employed ${ }^{16}$. Their results suggest that a small amount of sfGFP can be expressed under the control of $\mathrm{P}_{\mathrm{LtetO} 1}$ even in the presence of the repressor TetR.

Construction of a shikimate biosynthetic pathway in E. coli. Because shikimate is an important intermediate in the aromatic amino acid pathway, only $1.35 \pm 0.13 \mathrm{mg} / \mathrm{L}$ shikimate was accumulated during batch fermentation of wild-type E. coli BW25113 (Table 1). Metabolic engineering strategies were therefore applied to increase shikimate accumulation. To avoid possible interference, AraC was deleted from the BW25113 genome. To decrease the secretion of acetate, the main by-product in most shikimate-producing strains, pta encoding the phosphate acetyltransferase was inactivated. In strain P-2, however, shikimate production was still low $(2.25 \pm 0.15 \mathrm{mg} / \mathrm{L})$. As has been demonstrated by a carbon flux distribution analysis in wild-type E. coli, the PTS system is the largest consumer of PEP, with the relative carbon flux directed to the shikimate pathway representing only $1.5 \%$ of consumed $\mathrm{PEP}^{17}$. To provide more PEP for shikimate synthesis, we therefore inactivated $p t s G$ encoding the IIBC component of the glucose-specific PTS system. As shown in Table 1, strain P-3 achieved a shikimate production of $62.41 \mathrm{mg} / \mathrm{L}$, indicating that the intracellular level of PEP is essential to this process. Because aroK encoding shikimate kinase I was selected as the target for the tunable switch, aroL encoding shikimate 


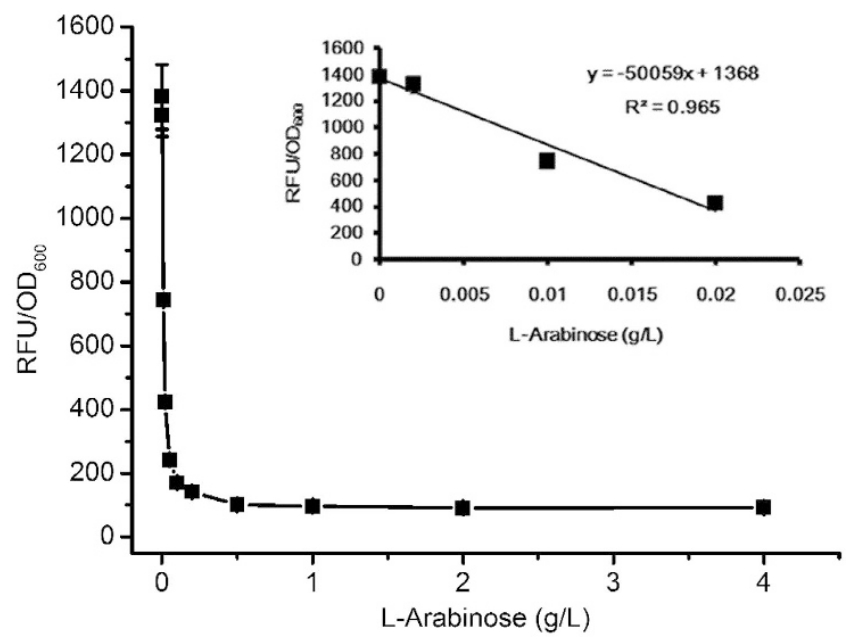

Figure 4. Relationship between concentration of the inducer $L$-arabinose and repression level of the tunable switch. Escherichia coli strains were cultured in $50 \mathrm{~mL}$ Luria-Bertani medium shaken at $250 \mathrm{rpm}$ and $37^{\circ} \mathrm{C}$. L-arabinose was added at $0 \mathrm{~h}$ and the fluorescence was determined at $12 \mathrm{~h}$. Error bars represent standard deviations based on three replicate fermentations.

\begin{tabular}{|l|c|c|c|c|c|}
\hline Strains & $\mathbf{C D W}^{\mathrm{b}}(\mathbf{g} / \mathrm{L})$ & $\begin{array}{c}\text { Specific growth } \\
\text { rate } \boldsymbol{\mu}(\mathbf{1} / \mathbf{h})\end{array}$ & $\begin{array}{c}\text { Glucose } \\
\text { consumption } \\
(\mathbf{g} / \mathrm{L})\end{array}$ & $\begin{array}{c}\text { Shikimate } \\
(\mathbf{m g} / \mathrm{L})\end{array}$ & Acetate $(\mathbf{g} / \mathrm{L})$ \\
\hline BW25113 & $4.25 \pm 0.22$ & $0.47 \pm 0.05$ & $14.63 \pm 0.76$ & $1.35 \pm 0.13$ & $9.25 \pm 0.37$ \\
\hline P-1 & $3.47 \pm \pm 0.14$ & $0.43 \pm 0.03$ & $14.02 \pm 0.43$ & $1.78 \pm 0.22$ & $7.16 \pm 0.33$ \\
\hline P-2 & $3.60 \pm 0.34$ & $0.41 \pm 0.05$ & $15.14 \pm 0.42$ & $2.25 \pm 0.15$ & $5.41 \pm 0.35$ \\
\hline P-3 & $3.20 \pm 0.21$ & $0.38 \pm 0.02$ & $14.82 \pm 0.43$ & $62.41 \pm 5.97$ & $1.51 \pm 0.28$ \\
\hline P-4 & $3.00 \pm \pm 0.21$ & $0.34 \pm 0.04$ & $14.51 \pm 0.98$ & $77.21 \pm 8.65$ & $0.47 \pm 0.08$ \\
\hline P-5 & $2.95 \pm 0.21$ & $0.37 \pm 0.05$ & $13.60 \pm 1.84$ & $105.03 \pm 11.32$ & $0.21 \pm 0.13$ \\
\hline P-6 & $3.93 \pm 0.16$ & $0.51 \pm 0.03$ & $9.80 \pm 0.76$ & $132.04 \pm 9.31$ & $0.10 \pm 0.01$ \\
\hline
\end{tabular}

Table 1. Fermentation parameters of strains engineered in shake flasks. ${ }^{a}$ Data represent the average of three samples taken from $36-\mathrm{h}$ batch cultures. ${ }^{\mathrm{b}}$ Cell dry weight.

kinase II was deleted to clearly characterise the function of this tunable switch. Compared with P-3, the shikimate production of P-4 increased by $23.71 \%$.

TrpR was next inactivated to eliminate the transcription regulation of genes involved in the shikimate pathway ${ }^{18,19}$, which further increased the production of shikimate to $105.03 \mathrm{mg} / \mathrm{L}$ (Table 1). In E. coli, pyruvate kinase isoenzymes encoded by $p y k F$ and $p y k A$ genes can catalyse the formation of pyruvate and Mg-ATP from PEP and $\mathrm{Mg}-\mathrm{ADP}$ in the presence of potassium ${ }^{6}$. Of these two pyruvate kinase isoenzymes, PykF contributes the most pyruvate kinase activity, and knock out of $p y k F$ has been reported to improve the production yield of shikimate ${ }^{20,21}$. As indicated in Table 1, P-6 with a deletion of $p y k F$ produced $132.04 \mathrm{mg} / \mathrm{L}$ shikimate. Surprisingly, the growth of P-6, as indicated by biomass and the specific growth rate, was recovered compared with that of P-5. Co-inactivation of PykF and the PTS system in E. coli, which has been reported to increase carbon flux from PEP into the TCA cycle through oxaloacetate ${ }^{22}$, may be responsible for the comparable growth of P-6 and wild-type BW25113. In addition, the genetic modification from BW25113 to P-6 was accompanied by a 9.25-0.10 g/L decrease in the secretion of acetate.

To further increase shikimate production and enhance E4P intracellular levels, $a r o G^{F B R}$ and $t k t A$, respectively encoding feedback-resistant 3-deoxy-D-arabinoheptulosonate-7-phosphate synthase and transketolase, were co-overexpressed in a low-copy plasmid, pCL1920. In addition, aroE, aroD and aroB, encoding dehydroshikimate reductase, 3-dehydroquinate dehydratase and 3-dehydroquinate synthase separately, were overexpressed in pUC19, which was compatible with pCL1920. Two recombinant plasmids, pF-1 and pF-12, were then simultaneously transformed into strain P-6 to generate P-8. In batch cultivation, strain P-8 exhibited tardy growth for $12 \mathrm{~h}$ and then entered into the logarithmic phase (Fig. 5). This long lag phase was possibly owing to the metabolic burden generated by the two recombinant plasmids. After $54 \mathrm{~h}$ batch cultivation, P- 8 produced $1.73 \mathrm{~g} / \mathrm{L}$ shikimate, which was 13.11 -fold higher than that of P-6, thus indicating that overexpression of the key enzymes in the shikimate pathway is necessary for shikimate production in E. coli.

Conditional repression of AroK with the tunable switch. By Red recombination, the tunable switch was integrated into the chromosome of P-6 to replace the original promoters, $\mathrm{P}_{\mathrm{aroK} 1}$ and $\mathrm{P}_{\text {aroK2 } 2}$. As a result, recombinant strain P-7 was obtained, which was then transformed with plasmids pF-1 and pF-12 to generate P-9. To explore the optimal time point for supplementation with inducer, L-arabinose was variously added to the 


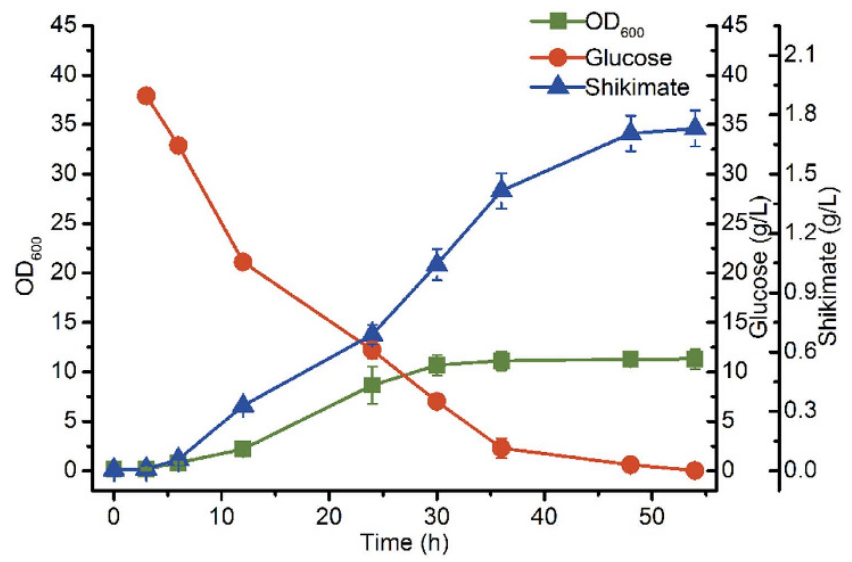

Figure 5. Batch cultivation of Escherichia coli P-8 in a 300-mL shake flask. Error bars represent standard deviations based on three replicate fermentations.
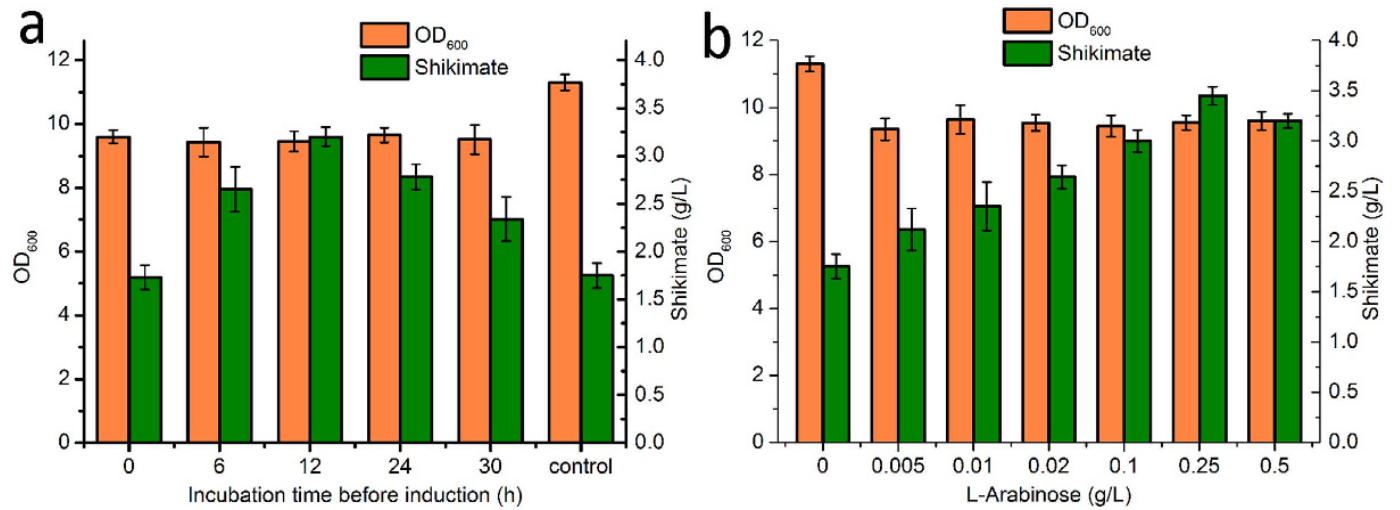

Figure 6. Exploration of the optimal supplementation time point and concentration of L-arabinose for shikimate production. (a) The optimal time point for supplementation with L-arabinose. The control strain was P-9 without L-arabinose. (b) The optimal supplemental concentration of L-arabinose. Error bars represent standard deviations based on three replicate experiments. Escherichia coli strains were cultured in $50 \mathrm{~mL}$ fermentation medium shaken at $250 \mathrm{rpm}$ and $37^{\circ} \mathrm{C}$, and shikimate production was determined at $54 \mathrm{~h}$.

medium at $0,6,12,24$ and $30 \mathrm{~h}$. Compared with the P-9 strain without L-arabinose, the other five P-9 strains with different supplemental time points of L-arabinose all exhibited slightly poorer growth (Fig. 6a), possibly because of decreased carbon flow from the downstream aromatic amino acid pathway. After supplementation with L-arabinose, however, higher shikimate accumulation was also observed. In particular, the strains supplemented with L-arabinose at $12 \mathrm{~h}$ exhibited the highest shikimate production, $3.22 \mathrm{~g} / \mathrm{L}$. In addition, only a slight increase was observed in shikimate production when $\mathrm{L}$-arabinose was added at $0 \mathrm{~h}$, probably the result of the low activity of AroK at the early phase. We next investigated the optimal supplemental concentration of $\mathrm{L}$-arabinose. Seven concentrations ranging from 0 to $0.5 \mathrm{~g} \mathrm{~L}^{-1}$ were selected and added to the medium at $12 \mathrm{~h}$ during batch fermentation of P-9 (Fig. 6b). Shikimate production increased from 1.75 to $3.45 \mathrm{~g} / \mathrm{L}$ as the concentration of L-arabinose was increased from 0 to $0.25 \mathrm{~g} / \mathrm{L}$. When the concentration of L-arabinose was further increased to $0.5 \mathrm{~g} / \mathrm{L}$, however, a slight reduction in the shikimate titre was recorded. Because shikimate production was affected by many factors, quantitative real-time reverse transcription PCR (qRT-PCR) was carried out to directly determine the relationship between the transcription level of aroK and the concentration of supplemental $\mathrm{L}$-arabinose. Compared with P-8 lacking the tunable switch, the relative aroK transcription of P-9 decreased from 1.05 to 0.09 as the concentration of L-arabinose was increased from 0 to $2.00 \mathrm{~g} / \mathrm{L}$ (Supplementary Fig. S1). This result was consistent with the relative fluorescence intensity shown in Fig. 4, thus demonstrating that the tunable switch was relatively stable when applied to different target genes.

In previously engineered shikimate-producing strains, aroK and aroL were permanently knocked out to block the aromatic amino acid biosynthetic pathway. Combined with other genetic modifications, high shikimate accumulation could thereby be achieved, but supplementation with various aromatic compounds was needed to maintain normal growth of the host $\operatorname{strain}^{6,7,23}$. Conditional interruption approaches that can maintain high expression of these genes until adequate cell mass is accumulated are consequently a promising strategy. In this study, we also constructed a recombinant strain, P-11, with an aroK deletion. In batch cultivation, P-11 exhibited normal growth and glucose consumption in medium containing aromatic compounds (Supplementary Fig. S2). After $54 \mathrm{~h}$, this 


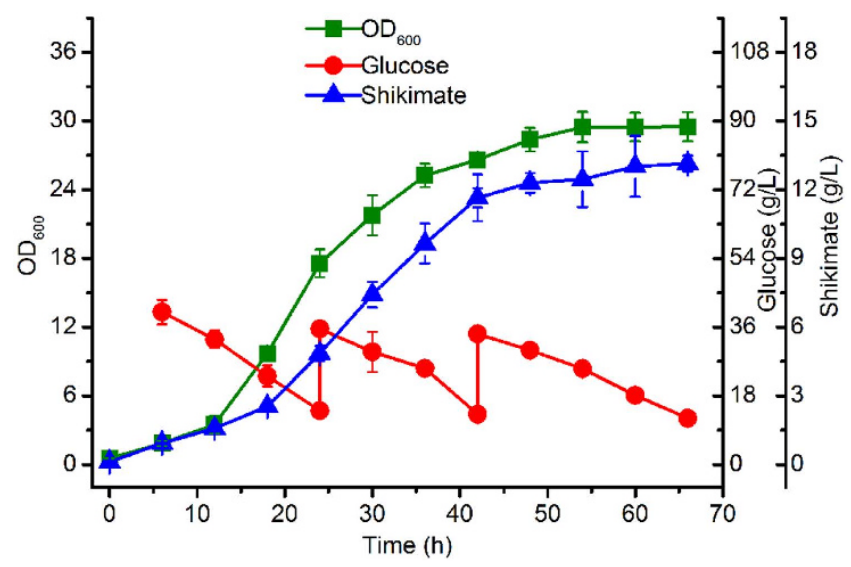

Figure 7. Fed-batch fermentation of P-9 in a 5-L fermentor. Error bars represent standard deviations based on three measurements.

strain produced $3.30 \mathrm{~g} / \mathrm{L}$ shikimate. As the aroK gene was deleted in P-11, the downstream pathway of shikimate was completely blocked, which may result in only a slight increasing of shikimate production for P-9 compared to that of P-11. However, supplement of aromatic compounds was indispensable for the culture of P-11, which indicated decreasing the expression level of aroK by the tunable switch was more effective and cost-saving than direct deletion of aroK in E. coli shikimate biosynthesis.

In 2014, Soma et al. constructed a metabolic toggle switch to regulate citrate synthase in E. coli. By switching gltA off and blocking the TCA cycle, the isopropanol production titre and yield were improved up to 3.7 and 3.1 times, respectively ${ }^{24}$. Using this strategy, the same group individually performed conditional repression of $g l p K, t p i A$ and $g a p A$, which are involved in glycerol metabolism, and improved the production titre and yield of 3 -hydroxypropionic acid by 80 and $94 \%$, respectively ${ }^{25}$. Compared with the complete pathway shutdown accomplished using their metabolic toggle switch, the tunable switch in this study can repress a target gene at different levels. Although no extra aromatic amino acids were added to the medium, strain P-9 exhibited no significant growth defects.

Finally, we compared the by-products of P- 8 and P-9 generated in batch fermentation. As shown in Supplementary Fig. S3, the acetate, lactate and pyruvate secreted by P-8 and P-9 were similar. After the introduction of the tunable switch, aroK transcription decreased, which led to the lower accumulation of three aromatic amino acids of P-9 compared with P-8. Moreover, strain P-9 exhibited higher quinate production than P-8. In E. coli, quinate is generated from 3-dehyroquinic acid catalysed by shikimate dehydrogenase isozymes YdiB and AroE. These two enzymes can also mediate the conversion of 3-dehydroshikimate to shikimate. As the reaction from 3-dehyroquinic acid to shikimate is reversible, the partial blockage of the downstream pathway and accumulation of shikimate in P-9 may have led to alteration of the reaction equilibrium. As a result, more quinate was secreted by $\mathrm{P}-9^{26,27}$.

Fermentation of strain P-9 in a 5-L fermentor. To evaluate the shikimate production potential of strain P-9, fed-batch fermentation was performed in a 5-L fermentor (Fig. 7). This strain exhibited a 12-h lag growth phase. During this period, shikimate also accumulated at low levels. Cell growth then entered the exponential phase. In the middle of the exponential phase, $0.25 \mathrm{~g} / \mathrm{L} \mathrm{L}$-arabinose was added. After $18 \mathrm{~h}$, the production of shikimate began to increase rapidly. In the P-9 strain, shikimate accumulation was growth dependent. The maximum shikimate accumulation, $13.15 \mathrm{~g} / \mathrm{L}$, was achieved at the maximum $\mathrm{OD}_{600}$ (29.52). Most importantly, P-9 is a non-auxotrophic shikimate-synthesising strain, with no aromatic compounds needed, thus providing a new avenue for the construction of a promising, economical, industrial shikimate-producing strain.

Other genetic strategies besides metabolic toggle switches have been used to manipulate protein expression levels to balance metabolic fluxes or down-regulate essential genes where a knock-out approach is not applicable. For example, an artificial noncoding small RNA (sRNA) library has been used to isolate and characterise recombinant $E$. coli strains with different repression levels of the endogenous genes ompF and $f l i C^{28}$. The clustered regularly interspaced short palindromic repeats (CRISPR)/Cas system is a powerful tool that has also been shown to perform highly selective transcriptional modulation. By using single-guide RNA targeting different regions of target genes, various repression levels can be achieved ${ }^{29}$. In E. coli, the SsrA tag is an 11-amino acid peptide that can render the target protein susceptible to tail-specific protease ${ }^{30}$. By adding an SsrA tag to the $\mathrm{C}$ terminus of TyrA, Doroshenko et al. constructed an L-phenylalanine-producing tyrosine-prototrophic E. coli strain with unstable TyrA. In M9 medium, this strain exhibited higher L-phenylalanine accumulation and a 10-fold lower Tyr/Phe ratio than the control strain with wild-type $\mathrm{TyrA}^{31}$. To ensure proper use of cellular resources, however, the expression of genes encoding enzymes at specific time points must also be controlled to the appropriate level. Compared with the aforementioned strategies, our tunable switch can achieve different repression levels via the direct addition of inducer with different concentrations at different times. 


\begin{abstract}
Methods
Bacterial strains. All strains, plasmids and primers used in this study are listed in Supplementary Tables S1-S3, respectively. Escherichia coli DH5 $\alpha$ was used as the host for recombinant DNA manipulation, and E. coli BW25113 was used to construct a basic shikimate-producing strain.
\end{abstract}

Gene deletion. The $a r a C$ gene encoding an arabinose operon regulatory protein was deleted by the traditional one-step inactivation method $^{32}$. Primers araC-F/araC-R and template plasmid pKD4 were used to obtain the linearised DNA. Primers araC-JF/araC-JR were then selected to verify the positive clones. After generation of strain P-1, the following genes were deleted in turn by the optimised one-step inactivation $\operatorname{method}^{33}: p t a$, $p t s G, \operatorname{aroL}, \operatorname{tr} p R$ and $p y k F$, respectively encoding phosphate acetyltransferase, fused phosphoenolpyruvate: carbohydrate phosphotransferase system IIBC components, shikimate kinase II, tryptophan pathway transcriptional repressor and pyruvate kinase I. To construct a control strain, aroK was also deleted. Primers pta-F/pta-R, ptsG-F/ptsG-R, aroL-F/aroL-R, trpR-F/trpR-R, pykF-F/pykF-R and aroK-F/aroK-R as well as genomic DNA of strains JW2294-1, JW1087-2, JW0379-1, JW4356-2, JW1666-3 and JW5947-1 were used to generate linearised DNA fragments with extended homologous sequences; in this way, recombinant strains P-2, P-3, P-4, P-5, P-6 and P-10 were successively obtained.

Plasmid construction. To simplify the whole construction process, generation of the tunable switch was divided into three modules. First, we used the genomic DNA of W3110 as a template and primers BAD-F/BAD-R to obtain the wild-type arabinose module containing AraC and $\mathrm{P}_{\mathrm{BAD}}$. $\mathrm{PCR}$ amplification with template plasmid pF-3 and primers T1-F/T1-R was then used to obtain the terminator BBa_B0014 (iGEM, http://parts.igem.org/). These two DNA fragments with 30-50 homologous arms were assembled and inserted into the BamHI site of pUC19 to generate plasmid pF- 4 by the enzymatic assembly strategy ${ }^{34}$. To construct module 2 containing regulatory protein TetR, promoter $\mathrm{P}_{\mathrm{LtetO} 1}$ and terminator BBa_B0015 (iGEM), we used template plasmids pwtCas9 and pF-5 and primers tetR-F/tetR-R, ptetO1-F/ptetO1-R and T2-F/T2-R to obtain these three DNA fragments. Next, homologous arms were added to the original tetR gene using primers tetR-YF/tetR-R to generate a new fragment, sTetR. sTetR, promoter $\mathrm{P}_{\mathrm{LtetO} 1}$ and terminator BBa_B0015 were then assembled and inserted into the Bam HI site of pUC19 to generate plasmid pF-6. Similarly, fragments of sfGFP and terminator BBa_J61048 (iGEM) with homologous arms were obtained by separately using template plasmids pF-7 and pF-8 and primers gfp-FC/gfp-R and T3-F/T3-R. These two fragments were assembled into pUC19 to generate plasmid pF-9. Finally, primers $1-\mathrm{F} / 1-\mathrm{R}, 2-\mathrm{F} / 2-\mathrm{R}$ and $3-\mathrm{CF} / 3-\mathrm{R}$ and templates $\mathrm{pF}-4, \mathrm{pF}-6$ and $\mathrm{pF}-9$ were used to obtain three modules with homologous arms that were inserted into the BamHI site of pUC19 to construct pF-2 by the enzymatic assembly strategy.

To obtain DNA fragments of $a r o E$, aroD and $a r o B$ by PCR, the genome of BW25113 was selected as a template, with aroE-F/aroE- $\mathrm{R}$, aroD-F/aroD-R and aroB-F/aroB-R respectively used as primers. Promoter $\mathrm{P}_{\mathrm{J} 23100}(\mathrm{iGEM})$ was then assembled with $\operatorname{trpE}$ by PCR using the original aroE fragment as a template and aroE-YF/aroE-R as primers. In addition, the fragment of terminator BBa_B0015 with homologous arms was generated with template plasmid pF-5 and primers T2-NF/T2-NR. The construction of pF-1 was carried out in three steps. First, $\mathrm{pF}-10$ was generated by assembling aroE and aroD into $\mathrm{pUC19}$, and pF-11 was generated by assembling aroB and BBa_B0015 into pUC19. Plasmids pF-10 and pF-11 were then used as templates and aroE-YF/ED-R and BT-F/ T2-NR as primers to obtain the two fragments, $\mathrm{P}_{\mathrm{J} 23100^{-}}$aroE-aroD and aroB- $\mathrm{T}_{\mathrm{BBa} \_ \text {B0015 }}$ with homologous arms. Finally, these two fragments were assembled into pUC19 to generate pF-1.

The aro $G^{F B R}-t k t A$ operon was generated using previously constructed pTAT and primers AT-NF/AT-NR. This fragment was inserted into pCL1920 by BamHI/XbaI double-digestion and T4 ligase-based ligation to generate plasmid pF-12.

Replacement of the original aroK promoter by the tunable switch. To improve the integration efficiency of the tunable switch, the chloramphenicol-resistance gene was selected as a marker. PCR using Cm-F/ $\mathrm{Cm}-\mathrm{R}$ and SSD-F/SSD-R as primers and plasmids $\mathrm{pKD} 3$ and $\mathrm{pF}-2$ as templates was used to generate two respective DNA fragments, cat and the tunable switch, with 30-50 arms homologous to each other. These two fragments were assembled into pUC19 to generate $\mathrm{pF}-13$, which then served as a template plasmid with primers TMTS-F/ TMTS-R to yield the final integration fragment. Red recombination was applied to accomplish this integration, and positive clones were verified by primers TMTS-JF/TMTS-JR. The resulting strain, P-7, was transformed with pF-1 and $\mathrm{pF}-12$ to generate $\mathrm{P}-9$. As a control, $\mathrm{pF}-1$ and $\mathrm{pF}-12$ were also transformed into P-10 to generate strain P-11.

qRT-PCR. Total mRNA of samples was extracted with an RNA Simple Total RNA kit (Tiangen, Beijing, China). Reverse transcription was performed using random 6-mer and oligo dT primers with a PrimeScript RT reagent kit (Takara, Dalian, China). The qRT-PCR was carried out with SYBR Premix Ex Taq II (Takara) following the LightCycler 480 RT-PCR System protocol (Roche, Basel, Switzerland). The qRT-PCR analysis was repeated three times for each sample. The qRT-PCR primers are listed in Supplementary Table S3.

Growth conditions. Strains for cloning and inoculation were grown in Luria-Bertani (LB) medium (10 g/L tryptone, $5 \mathrm{~g} / \mathrm{L}$ yeast extract, and $10 \mathrm{~g} / \mathrm{L} \mathrm{NaCl})$ at $37^{\circ} \mathrm{C}$ for $8-12 \mathrm{~h}$. Ampicillin $(100 \mathrm{mg} / \mathrm{L})$, chloramphenicol $(17 \mathrm{mg} / \mathrm{L})$, kanamycin $(25 \mathrm{mg} / \mathrm{L})$, spectinomycin $(50 \mathrm{mg} / \mathrm{L})$, or tetracycline $(20 \mathrm{mg} / \mathrm{L})$ was incorporated into the medium when necessary. SOB medium $(20 \mathrm{~g} / \mathrm{L}$ tryptone, $5 \mathrm{~g} / \mathrm{L}$ yeast extract, $0.5 \mathrm{~g} / \mathrm{L} \mathrm{NaCl}, 2.5 \mathrm{mM} \mathrm{KCl}$, and $10 \mathrm{mM} \mathrm{MgCl} 2$ ) was used for gene deletion. Isopropyl $\beta$-D-1-thiogalactopyranoside was added at a final concentration of $0.2 \mathrm{mM}$. The fermentation medium was as described in a previous study with the three aromatic amino acids excluded ${ }^{6}$. For the cultivation of $\mathrm{P}-11$, however, the three aromatic amino acids were included. A single clone was pre-cultured in $5 \mathrm{~mL} \mathrm{LB}$ medium at $37^{\circ} \mathrm{C}$ on a rotary shaker at $250 \mathrm{rpm}$ overnight. One milliliter of overnight cells were inoculated into $50 \mathrm{~mL} \mathrm{LB}$ medium and cultured for $8-12 \mathrm{~h}$, with $10 \%(\mathrm{v} / \mathrm{v})$ seed cultures 
subsequently incubated into $50 \mathrm{~mL}$ fermentation medium at $37^{\circ} \mathrm{C}$ and $250 \mathrm{rpm}$. For fed-batch fermentation, a stirred 5-L glass vessel with the BioFlo310 modular fermentor system (New Brunswick Scientific, Edison, NJ, USA) was used. Single clones were pre-cultured in $50 \mathrm{~mL} \mathrm{LB}$ medium at $37^{\circ} \mathrm{C}$ on a rotary shaker at $250 \mathrm{rpm}$ overnight. Five milliliters of overnight cells were inoculated into $200 \mathrm{~mL}$ fermentation medium and cultured for $8-12 \mathrm{~h} ; 10 \%(\mathrm{v} / \mathrm{v})$ seed cultures were then added to the fermentor containing $3.5 \mathrm{~L}$ fermentation medium. Samples were taken at 6 -h intervals. When the glucose concentration in the medium fell below $10 \mathrm{~g} / \mathrm{L}$, the medium was supplemented with a feeding solution containing $500 \mathrm{~g} / \mathrm{L}$ glucose to reach a final concentration of approximately $30 \mathrm{~g} / \mathrm{L}$. The dissolved oxygen concentration was maintained above $30 \%$ air saturation, and the $\mathrm{pH}$ was kept at 7.0 through $\mathrm{NH}_{4} \mathrm{OH}$ buffering.

Analytical methods. Cell growth was monitored at $\mathrm{OD}_{600}$ with a spectrophotometer (Shimadzu, Kyoto Japan). Glucose concentration was determined with a glucose biosensor (SBA-40C; Biology Institute of Shandong Academy of Sciences, Jinan, China). Shikimate and quinate were quantitatively analysed on a high-performance liquid chromatography (HPLC) system (Shimadzu) equipped with a HPX-87H column $(300 \mathrm{~mm} \times 7.8 \mathrm{~mm} ; 9 \mu \mathrm{m}$; Bio-Rad, Hercules, CA, USA) maintained at $50^{\circ} \mathrm{C}$. The mobile phase was $5 \mathrm{mM} \mathrm{H}_{2} \mathrm{SO}_{4}$ at a flow rate of $0.6 \mathrm{~mL} / \mathrm{min}$. Peaks were detected using a photodiode array detector at $210 \mathrm{~nm}$. For detection of acetate, lactate, and pyruvate, we used an HPLC system with a refractive index detector (RID-10A) (Shimadzu) and a HPX-87H ion exclusion column (Bio-Rad) at $65^{\circ} \mathrm{C}$. The mobile phase was $5 \mathrm{mM} \mathrm{H}_{2} \mathrm{SO}_{4}$ at a flow rate of $0.6 \mathrm{~mL} / \mathrm{min}$. Accumulation of L-phenylalanine, L-tyrosine, and L-tryptophan in strains P-8 and P-9 was determined with a Venusil AA analysis kit (Bonna-Agela Technologies, Tianjin, China). The fluorescence of recombinant strains was determined in 96-well microtitre plates as described previously ${ }^{33}$.

\section{References}

1. Kim, C. U. et al. Influenza neuraminidase inhibitors possessing a novel hydrophobic interaction in the enzyme active site: design, synthesis, and structural analysis of carbocyclic sialic acid analogues with potent anti-influenza activity. J Am Chem Soc. 119, 681-690 (1997).

2. Kramer, M. et al. Metabolic engineering for microbial production of shikimic acid. Metab Eng. 5, 277-283 (2003).

3. Adachi, O., Ano, Y., Toyama, H. \& Matsushita, K. High shikimate production from quinate with two enzymatic systems of acetic acid bacteria. Biosci Biotechnol Biochem. 70, 2579-2582 (2006).

4. Ghosh, S. \& Banerjee, U. C. Generation of aroE overexpression mutant of Bacillus megaterium for the production of shikimic acid. Microb Cell Fact 14, 69 (2015).

5. Liu, X., Lin, J., Hu, H., Zhou, B. \& Zhu, B. Site-specific integration and constitutive expression of key genes into Escherichia coli chromosome increases shikimic acid yields. Enzyme Microb Technol. 82, 96-104 (2016).

6. Chen, X. et al. Metabolic engineering of Escherichia coli for improving shikimate synthesis from glucose. Bioresour Technol. 166, 64-71 (2014).

7. Escalante, A. et al. Metabolic engineering for the production of shikimic acid in an evolved Escherichia coli strain lacking the phosphoenolpyruvate: carbohydrate phosphotransferase system. Microb Cell Fact. 9, 21 (2010).

8. Cui, Y. Y., Ling, C., Zhang, Y. Y., Huang, J. \& Liu, J. Z. Production of shikimic acid from Escherichia coli through chemically inducible chromosomal evolution and cofactor metabolic engineering. Microb Cell Fact. 13, 21 (2014).

9. Gu, P., Yang, F., Kang, J., Wang, Q. \& Qi, Q. One-step of tryptophan attenuator inactivation and promoter swapping to improve the production of L-tryptophan in Escherichia coli. Microb Cell Fact 11, 30 (2012).

10. Yi, J., Draths, K. M., Li, K. \& Frost, J. W. Altered glucose transport and shikimate pathway product yields in E. coli. Biotechnol Prog. 19, 1450-1459 (2003)

11. Patnaik, R., Spitzer, R. G. \& Liao, J. C. Pathway engineering for production of aromatics in Escherichia coli: Confirmation of stoichiometric analysis by independent modulation of AroG, TktA, and Pps activities. Biotechnol Bioeng. 46, 361-370 (1995).

12. Lu, J. L. \& Liao, J. C. Metabolic engineering and control analysis for production of aromatics: Role of transaldolase. Biotechnol Bioeng. 53, 132-138 (1997).

13. Chandran, S. S. et al. Phosphoenolpyruvate availability and the biosynthesis of shikimic acid. Biotechnol Prog. 19, 808-814 (2003).

14. Knop, D. R. et al. Hydroaromatic equilibration during biosynthesis of shikimic acid. J Am Chem Soc. 123, 10173-10182 (2001).

15. Fernandez-Castane, A., Caminal, G. \& Lopez-Santin, J. Direct measurements of IPTG enable analysis of the induction behavior of E. coli in high cell density cultures. Microb Cell Fact. 11, 58 (2012).

16. Rogers, J. K. et al. Synthetic biosensors for precise gene control and real-time monitoring of metabolites. Nucleic Acids Res. 43, 7648-7660 (2015).

17. Flores, S., Gosset, G., Flores, N., de Graaf, A. A. \& Bolivar, F. Analysis of carbon metabolism in Escherichia coli strains with an inactive phosphotransferase system by ${ }^{13} \mathrm{C}$ labeling and NMR spectroscopy. Metab Eng. 4, 124-137 (2002).

18. Berry, A. Improving production of aromatic compounds in Escherichia coli by metabolic engineering. Trends Biotechnol. 14, 250-256 (1996)

19. Gunsalus, R. P. \& Yanofsky, C. Nucleotide sequence and expression of Escherichia coli trpR, the structural gene for the trp aporepressor. Proc Natl Acad Sci USA 77, 7117-7121 (1980).

20. Rodriguez, A. et al. Constitutive expression of selected genes from the pentose phosphate and aromatic pathways increases the shikimic acid yield in high-glucose batch cultures of an Escherichia coli strain lacking PTS and pykF. Microb Cell Fact. 12, 86 (2013).

21. Licona-Cassani, C. et al. Inactivation of pyruvate kinase or the phosphoenolpyruvate: sugar phosphotransferase system increases shikimic and dehydroshikimic acid yields from glucose in Bacillus subtilis. J Mol Microbiol Biotechnol. 24, 37-45 (2014).

22. Meza, E., Becker, J., Bolivar, F., Gosset, G. \& Wittmann, C. Consequences of phosphoenolpyruvate:sugar phosphotranferase system and pyruvate kinase isozymes inactivation in central carbon metabolism flux distribution in Escherichia coli. Microb Cell Fact. 11, 127 (2012).

23. Ths, K. M. \& Knop, D. R. \& Frost, J. W. Shikimic Acid and Quinic Acid: Replacing isolation from plant sources with recombinant microbial biocatalysis. J Am Chem Soc. 121 (1999).

24. Soma, Y., Tsuruno, K., Wada, M., Yokota, A. \& Hanai, T. Metabolic flux redirection from a central metabolic pathway toward a synthetic pathway using a metabolic toggle switch. Metab Eng 23, 175-184 (2014).

25. Tsuruno, K., Honjo, H. \& Hanai, T. Enhancement of 3-hydroxypropionic acid production from glycerol by using a metabolic toggle switch. Microb Cell Fact. 14, 155 (2015).

26. Juminaga, D. et al. Modular engineering of L-tyrosine production in Escherichia coli. Appl Environ Microbiol. 78, 89-98 (2012).

27. Kim, B. G., Jung, W. D., Mok, H. \& Ahn, J. H. Production of hydroxycinnamoyl-shikimates and chlorogenic acid in Escherichia coli: production of hydroxycinnamic acid conjugates. Microb Cell Fact. 12, 15 (2013). 
28. Sharma, V., Yamamura, A. \& Yokobayashi, Y. Engineering artificial small RNAs for conditional gene silencing in Escherichia coli. ACS Synth Biol. 1, 6-13 (2012).

29. Cress, B. F. et al. CRISPathBrick: Modular combinatorial assembly of type II-A CRISPR arrays for dCas9-mediated multiplex transcriptional repression in E. coli. ACS Synth Biol. 4, 987-1000 (2015).

30. Gottesman, S., Roche, E., Zhou, Y. \& Sauer, R. T. The ClpXP and ClpAP proteases degrade proteins with carboxy-terminal peptide tails added by the SsrA-tagging system. Genes Dev. 12, 1338-1347 (1998).

31. Doroshenko, V. G. et al. Construction of an L-phenylalanine-producing tyrosine-prototrophic Escherichia coli strain using tyrA ssrA-like tagged alleles. Biotechnol Lett. 32, 1117-1121 (2010).

32. Datsenko, K. A. \& Wanner, B. L. One-step inactivation of chromosomal genes in Escherichia coli K-12 using PCR products. Proc Natl Acad Sci USA 97, 6640-6645 (2000).

33. Li, M. et al. Extending homologous sequence based on the single gene mutants by one-step PCR for efficient multiple gene knockouts. Folia Microbiol (Praha) 57, 209-214 (2012).

34. Gibson, D. G. et al. Enzymatic assembly of DNA molecules up to several hundred kilobases. Nat Methods 6, 343-345 (2009).

35. Pedelacq, J. D., Cabantous, S., Tran, T., Terwilliger, T. C. \& Waldo, G. S. Engineering and characterization of a superfolder green fluorescent protein. Nat Biotechnol. 24, 79-88 (2006)

\section{Acknowledgements}

This research was financially supported by grants from the National Basic Research Program of China (2012CB725202), the National Natural Science Foundation of China (31370085), the China Postdoctoral Science Foundation Funded Project (2014M551894), Special Funds of Shandong Postdoctoral Innovation Project (201402026) and the Technology Development Plan (2015GSF121042).

\section{Author Contributions}

P.G. carried out most of the experiments. T.S. and Q.W. performed batch and fed-batch fermentation. Q.Q., P.G., Q.W. and Q.L. drafted the manuscript. Q.L. and Q.Q. conceived and supervised the study. All authors read and approved the final manuscript.

\section{Additional Information}

Supplementary information accompanies this paper at http://www.nature.com/srep

Competing financial interests: The authors declare no competing financial interests.

How to cite this article: $\mathrm{Gu}$, P. et al. Tunable switch mediated shikimate biosynthesis in an engineered nonauxotrophic Escherichia coli. Sci. Rep. 6, 29745; doi: 10.1038/srep29745 (2016).

(c) (i) This work is licensed under a Creative Commons Attribution 4.0 International License. The images or other third party material in this article are included in the article's Creative Commons license, unless indicated otherwise in the credit line; if the material is not included under the Creative Commons license, users will need to obtain permission from the license holder to reproduce the material. To view a copy of this license, visit http://creativecommons.org/licenses/by/4.0/ 